\title{
Evaluation of Radiation Dose and Image Quality using High-Pitch 70-kV Chest CT in Immunosuppressed Patients
}

\section{Evaluation der Strahlendosis und Bildqualität von High-Pitch-70-kV- CT-Untersuchungen des Thorax bei immunsupprimierten Patienten}

Authors

Ibrahim Yel, Simon S. Martin, Julian L. Wichmann, Lukas Lenga, Moritz H. Albrecht, Andreas M. Bucher, Benjamin Kaltenbach, Christoph Polkowski, Christian Booz, Thomas J. Vogl, Renate Maria Hammerstingl

\section{Affiliation}

Department of Diagnostic and Interventional Radiology, University-Hospital Frankfurt, Germany

Key words

thorax, CT, high-pitch CT, radiation dose,

immunosuppression

received 25.01 .2018

accepted 07.07.2018

\section{Bibliography}

DOI https://doi.org/10.1055/a-0668-6031

Published online: 4.9.2018

Fortschr Röntgenstr 2019; 191: 122-129

(c) Georg Thieme Verlag KG, Stuttgart · New York

ISSN 1438-9029

\section{Correspondence}

\section{Dr. Julian L. Wichmann}

Institut für Diagnostische und Interventionelle Radiologie, J.-W.-Goethe-Universität Frankfurt, Theodor-Stern-Kai 7, 60590 Frankfurt, Germany

Tel.: ++49/69/630187200

docwichmann@gmail.com

\section{ABSTRACT}

Purpose The aim of the study was to evaluate high-pitch $70-k V$ CT examinations of the thorax in immunosuppressed patients regarding radiation dose and image quality in comparison with 120-kV acquisition.

Materials and Methods The image data from 40 patients (14 women and 26 men; mean age: $40.9 \pm 15.4$ years) who received high-pitch 70-kV CT chest examinations were retrospectively included in this study. A control group $(n=40)$, matched by age, gender, BMI, and clinical inclusion criteria, had undergone standard $120-\mathrm{kV}$ chest CT imaging. All CT scans were performed on a third-generation dual-source CT unit. For an evaluation of the radiation dose, the CT dose index (CTDIvol), dose-length product (DLP), effective dose (ED), and size-specific dose estimates (SSDE) were analyzed in each group. The objective image quality was evaluated using signal-to-noise (SNR) and contrast-to-noise ratios
(CNR). Three blinded and independent radiologists evaluated subjective image quality and diagnostic confidence using 5-point Likert scales.

Results The mean dose parameters were significantly lower for high-pitch 70-kV CT examinations (CTDIvol, $2.9 \pm 0.9$ mGy; DLP, $99.9 \pm 31.0$ mGyxcm; ED, $1.5 \pm 0.6$ mSv; SSDE, 3.8 $\pm 1.2 \mathrm{mGy}$ ) compared to standard $120-\mathrm{kV}$ CT imaging (CTDIvol, $8.8 \pm 3.7 \mathrm{mGy}$; DLP, $296.6 \pm 119.3$ mGyxcm; ED, $4.4 \pm 2.1$ $\mathrm{mSv}$; SSDE, $11.6 \pm 4.4 \mathrm{mGy})(\mathrm{P} \leq 0.001)$. The objective image parameters (SNR: $7.8 \pm 2.1$ vs. $8.4 \pm 1.8$; CNR: $7.7 \pm 2.4$ vs. $8.3 \pm 2.8)(P \geq 0.065)$ and the cumulative subjective image quality $(4.5 \pm 0.4$ vs. $4.7 \pm 0.3)(p=0.052)$ showed no significant differences between the two protocols.

Conclusion High-pitch 70-kV thoracic CT examinations in immunosuppressed patients resulted in a significantly reduced radiation exposure compared to standard 120-kV CT acquisition without a decrease in image quality.

Key Points:

- Third-generation dual-source CT units enable high-pitch 70-kV CT examinations of the chest.

- High-pitch 70-kV CT examinations show a significantly reduced radiation dose compared to standard 120-kV CT examinations.

- High-pitch 70-kV CT examinations of the chest show comparable objective and subjective image quality.

- Subjectively deteriorated image noise and sharpness of 70-kV CT did not impact diagnostic confidence.

\section{Citation Format}

- Yel I, Martin SS, Wichmann JL et al. Evaluation of Radiation Dose and Image Quality using High-Pitch 70-kV Chest CT in Immunosuppressed Patients . Fortschr Röntgenstr 2019; 191: 122-129

\section{ZUSAMMENFASSUNG}

Ziel Das Ziel der Studie war, die Strahlendosis und Bildqualität von High-Pitch-70-kV-CT-Untersuchungen des Thorax bei immunsupprimierten Patienten im Vergleich zu 120-kV-CT-Bildgebung zu evaluieren.

Material und Methoden Die Bilddaten von 40 konsekutiven Patienten (14 Frauen und 26 Männer; Altersdurchschnitt 40,9 $\pm 15,4$ Jahre), die eine High-Pitch-70-kV-CT-Unter- 
suchung des Thorax erhielten, wurden retrospektiv ausgewertet. Eine gematchte Patientengruppe $(n=40)$ mit ähnlichem Alter, Geschlecht, BMI und klinischer Indikation, bei denen eine Standard-120-kV-CT-Untersuchung des Thorax durchgeführt wurde, diente als Kontrollgruppe. Alle CT-Aufnahmen erfolgten an einem Dual-Source-CT der dritten Generation. Zur Dosisabschätzung wurden der CT-Dosis-Index (CTDIvol), das Dosis-Längen-Produkt (DLP), die effektive Dosis (ED) und die größenspezifische Dosisabschätzung (SSDE) analysiert. Die objektive Bildqualität wurde mittels des Signal-RauschVerhältnisses (SNR) und des Kontrast-Rausch-Verhältnisses (CNR) ermittelt. Drei verblindete und unabhängige Radiologen evaluierten die subjektive Bildqualität und Diagnosesicherheit anhand von 5-Punkten-Likert-Skalen.

Ergebnisse Die mittleren Dosisparameter waren signifikant geringer für High-Pitch-70-kV-CT-Untersuchungen (CTDIvol

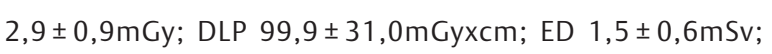
SSDE 3,8 $\pm 1,2 \mathrm{mGy}$ ) im Vergleich zu den Standard-120-kV-CTThorax-Untersuchungen (CTDIvol 8,8 $\pm 3,7 \mathrm{mGy}$; DLP 296,6 $\pm 119,3 \mathrm{mGyxcm}$; ED 4,4 $\pm 2,1 \mathrm{mSv}$; SSDE 11,6 $\pm 4,4 \mathrm{mGy}$ ) (alle $\mathrm{p} \leq 0,001$ ). Die objektiven Bildparameter (SNR 7,8 $\pm 2,1$ vs. 8,4 $\pm 1,8$; CNR $7,7 \pm 2,4$ vs. $8,3 \pm 2,8)(p \geq 0,065)$ sowie die kumulative subjektive Bildqualität $(4,5 \pm 0,4$ vs. $4,7 \pm 0,3)(p=0,052)$ zeigten keine signifikanten Unterschiede zwischen beiden CT-Untersuchungsprotokollen.

Schlussfolgerung High-Pitch-70-kV-CT-Untersuchungen des Thorax bei immunsupprimierten Patienten resultieren in einer deutlichen Reduktion der Strahlenbelastung im Vergleich zu Standard-120-kV-CT-Bildakquisition ohne eine Einschränkung der Bildqualität.

\section{Introduction}

Pulmonary infections are typically significantly more dangerous in immunosuppressed patients than in immunocompetent patients due to their atypical and prolonged course [1 - 3]. In light of the greater number of complications and the increased lethality, it is therefore often necessary to initiate appropriate and targeted therapy even prior to obtaining definitive pathogen confirmation which can take up to several days [4]. The most important part of primary evaluation is imaging [1]. Since conventional X-ray images do not allow definitive diagnosis in immunosuppressed patients due to the variable immune response and disease extent in many cases, high-resolution CT imaging of the lung is preferred for obtaining initial information regarding the etiology of the infection $[1,5]$.

The patient's radiation exposure accumulates with each examination, a fact that is relevant with an increasing duration of disease and progressive number of examinations particularly in this patient population which tends to be younger [6-8]. In light of the increased risk of cancer in the case of multiple examinations, the medical indication should be strictly reviewed and examination protocols and radiation exposure should be optimized.

The goal of this study was to evaluate high-pitch 70-kV CT examinations of the chest regarding radiation dose and image quality and to compare them with standard 120-kV CT examinations of the chest.

\section{Materials and Methods}

\section{Patient group}

The image data of 40 consecutive patients ( 14 women and 26 men; average age: $40.9 \pm 15.4$ years; age range: $20-84$ years; BMI: $26.5 \pm 2.6 \mathrm{~kg} / \mathrm{m}^{2}$ ) who underwent an unenhanced CT examination of the chest using a high-pitch 70-kV examination protocol at our radiology institute in the period from January 2017 to June 2017 were examined in this retrospective study. This protocol was defined as the standard protocol for immunosuppressed patients as of January 2017 based on the technical feasibility (lowest possible tube voltage) and the current research regarding dose reduction in CT examinations of the chest.

A matched group of 40 patients ( 14 women and 26 men; average age: $39.5 \pm 15.7$ years; age range: $19-86$ years; BMI: $27.2 \pm$ $3.6 \mathrm{~kg} / \mathrm{m}^{2}$ ) examined with the $120-\mathrm{kV}$ standard protocol prior to January 2017 served as the control group. These patients were matched with respect to age, sex, BMI, scan field length, and clinical indication criteria. 12 patients who first underwent a $120-\mathrm{kV}$ CT examination and then a high-pitch 70-kV CT examination were included in both the study group and the control group. $>$ Table 1 shows the patient characteristics.

The inclusion criteria were immunosuppressed patients with acquired, congenital, or induced immunodeficiency who underwent a clinically indicated CT examination of the chest without contrast administration. The exclusion criteria were examinations in patients younger than 18 years and CT examinations with significant motion or breathing artifacts not allowing evaluation of the secondary lobule, the last functional pulmonary structure morphologically visible on CT, and the pulmonary interstitium [9].

\section{CT examination protocol}

All examinations were performed on a third-generation dualsource CT unit (SOMATOM Force, Siemens Healthcare, Forchheim). The CT images were acquired in a supine position during deep inspiration. After acquisition of a topogram from the 7th cervical vertebra to the lower costal arch, the CT examination was performed in the craniocaudal view. Automatic modulation of the tube current adapted and optimized to the patient's habitus was performed for both examination protocols $(70 \mathrm{kV}$ and $120 \mathrm{kV}$ ) using suitable dose reduction software (Care Dose 4D, Siemens). The high-pitch 70-kV CT examination protocol included a pitch factor of 3.2 (turbo flash mode, Siemens) and a resulting table speed of $737 \mathrm{~mm} / \mathrm{s}$, while a pitch factor of 1.2 and a consequently slower table speed of $77 \mathrm{~mm} / \mathrm{s}$ were used for the $120-\mathrm{kV}$ standard protocol. A precise list of the CT parameters for both examination protocols is shown in $\triangleright$ Table 2 . 
- Table 1 Patient characteristics.

\begin{tabular}{|l|l|l|}
\hline & high-pitch 70-kV & standard 120-kV \\
\hline number of patients & 40 & 40 \\
\hline men/women & $26 / 14$ & $26 / 14$ \\
\hline age in years (range) & $40.9 \pm 15.4$ & $39.5 \pm 15.7$ \\
& $(20-84)$ & $(19-86)$ \\
\hline BMI in $\mathrm{kg} / \mathrm{m}^{2}$ (range) & $26.5 \pm 2.6$ & $27.2 \pm 3.6$ \\
& $(21.4-33.6)$ & $(22.7-33.8)$ \\
\hline
\end{tabular}

Image reconstruction from the raw datasets was performed using dedicated iterative 3 rd generation image reconstruction (ADMIRE, Siemens, strength 3 of 5 ) with a soft tissue kernel (Br36) and lung kernel (BI57) in axial, coronal, and sagittal views with a slice thickness of $1.0 \mathrm{~mm}$ and an increment of $1.0 \mathrm{~mm}$.

\section{Evaluation of radiation dose}

The $C T$ dose index $\left(C T D I_{\text {vol }}\right)$ and dose length product (DLP) were evaluated after every examination on the basis of the patient protocol. According to the general guidelines on the quality criteria for CT examinations, a conversion factor $(k)$ of 0.0147

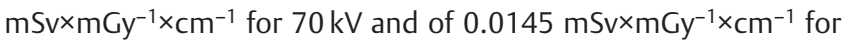
$120 \mathrm{kV}$ examinations was used to estimate the effective dose (ED) based on the following formula $[10,11]$.

$$
\mathrm{ED}=\mathrm{DLP} \times \mathrm{k}
$$

The size-specific dose estimate (SSDE) was calculated according to the recommendations of the American Association of Physicists in Medicine [12]. A conversion factor based on a 32-cm CTDI phantom and the sum of the lateral and anterior-posterior diameter ( $\left(_{\text {size }}^{32}\right)$ was multiplied by $\mathrm{CTDI}_{\mathrm{vol}}$ :

$\mathrm{SSDE}=f_{\text {size }}^{32} \times C \mathrm{CTDI}_{\mathrm{vol}}$

\section{Objective image quality}

The image quality was objectively evaluated based on the signalto-noise ratio (SNR) and contrast-to-noise ratio (CNR). Circular regions of interest (ROI) (size: $150 \mathrm{~mm}^{2}$ ) were placed in the descending aorta, in the lung parenchyma, in the autochthonous back musculature and in the mediastinal fat at the level of the tracheal bifurcation. The measurements were performed in the soft tissue window (aorta, Br36 kernel) and lung window (lung, BI57 kernel). The average attenuation in Hounsfield units (HU) and the standard deviation (SD) were noted for all measurements. In concordance with previous studies, the SNR and CNR values for the aorta and the lung parenchyma were determined on the basis of the following calculations $[13,14]$ :

SNR $=\frac{\mid \mathrm{HU} \text { (Aorta; Lunge) } \mid}{\text { SD (Mediastinales Fett) }}$

$\mathrm{CNR}=\frac{\mid \mathrm{HU} \text { (Aorta; Lunge) }-\mathrm{HU} \text { (Rückenmuskulatur) } \mid}{\mathrm{SD} \text { (Mediastinales Fett) }}$
- Table 2 CT acquisition parameters.

\begin{tabular}{|l|l|l|}
\hline & high-pitch 70-kV & standard 120-kV \\
\hline CT unit & $\begin{array}{l}3 \text { rd generation } \\
\text { DSCT }\end{array}$ & $\begin{array}{l}\text { 3 rd generation } \\
\text { DSCT }\end{array}$ \\
\hline $\begin{array}{l}\text { examination } \\
\text { mode }\end{array}$ & SECT & SECT \\
\hline tube voltage & $70 \mathrm{kV}$ & $120 \mathrm{kV}$ \\
\hline tube current & $200 \mathrm{ref} . \mathrm{mAs}$ & $110 \mathrm{ref} . \mathrm{mAs}$ \\
\hline pitch & 3.2 & 1.2 \\
\hline table speed & $737 \mathrm{~mm} / \mathrm{s}$ & $77 \mathrm{~mm} / \mathrm{s}$ \\
\hline rotation time & $0.25 \mathrm{~s}$ & $0.25 \mathrm{~s}$ \\
\hline collimation & $2 \times 192 \times 0.6 \mathrm{~mm}$ & $192 \times 0.6 \mathrm{~mm}$ \\
\hline slice thickness & $1 \mathrm{~mm}$ & $1 \mathrm{~mm}$ \\
\hline increment & $1 \mathrm{~mm}$ & $1 \mathrm{~mm}$ \\
\hline DSCT = dual-source CT; SECT single-energy CT. & \\
\hline
\end{tabular}

\section{Subjective image quality}

Three radiologists with three to five years of CT lung imaging experience subjectively evaluated the CT images. The rating was performed on certified diagnostic screens (RadiForce RX240; Eizo, Ishikawa, Japan) using the Centricity PACS Viewer software (GE Healthcare, Milwaukee, USA). All datasets were randomized and anonymized with respect to patient identity and scan setting. The radiologists were allowed to scroll through all orthogonal reconstructions (axial, coronal, and sagittal) and to adjust window settings at their discretion. The currently established settings for the lung window (width $2000 \mathrm{HU} /$ center $-500 \mathrm{HU}$ ) and soft tissue window (width $400 \mathrm{HU} /$ center $50 \mathrm{HU}$ ) were preset. The general image quality (from 1 = poor image quality to $5=$ excellent image quality), the image noise (from $1=$ extensive image noise to $5=$ no image noise detectable), the image sharpness (from 1 = extremely unsharp to 5 = excellent image sharpness), the presence of motion artifacts (from $1=$ pronounced motion artifacts to $5=$ no motion artifacts) and the subjective diagnostic reliability (from $1=$ no diagnostic significance to 5 = high diagnostic significance) were evaluated using a 5-point Likert scale.

\section{Statistical analysis}

All statistical analyses were performed using suitable statistics programs (GraphPad Prism Version 7.0, GraphPad Software, La Jolla, USA and SPSS Version 21, IBM SPSS Statistics, Armonk, USA). The results of the measurements and evaluations were provided as mean values with the corresponding standard deviations (SD). The Gaussian distribution of all data was tested using the Kolmogorov-Smirnov test and the data was analyzed using the Mann-Whitney $U$-test in consideration of the fact that some patients were included in both groups. P-values $<0.05$ were considered statistically significant.

Agreement between raters was determined with the intraclass correlation coefficient (ICC) and was evaluated as follows [15]: ICC 
- Table 3 Dosimetric parameters.

\begin{tabular}{|l|c|c|c|}
\hline & high-pitch 70-kV & standard 120-kV & p-value \\
\hline CTDI $_{\text {vol }}$ (mGy) & $2.9 \pm 0.9(1.9-3.8)$ & $8.8 \pm 3.7(1.6-22.3)$ & $\mathrm{p}<0.001$ \\
\hline DLP (mGy $\times \mathrm{cm})$ & $99.9 \pm 31.0(58.9-165.5)$ & $296.6 \pm 119.3(68.5-640.9)$ & $\mathrm{p}<0.001$ \\
\hline ED (mSv) & $1.5 \pm 0.6(0.9-2.4)$ & $4.4 \pm 2.1(1.0-9.4)$ & $\mathrm{p}<0.001$ \\
\hline SSDE (mGy) & $3.8 \pm 1.2(2.5-5.1)$ & $11.6 \pm 4.4(2.4-26.8)$ & $\mathrm{p}<0.001$ \\
\hline Scan field length $(\mathrm{cm})$ & $31.4 \pm 4.2(25.0-40.5)$ & $30.7 \pm 6.5(24.5-41.9)$ & $\mathrm{p}=0.077$ \\
\hline
\end{tabular}

$<0.40=$ poor agreement, ICC $0.40-0.59=$ moderate agreement, ICC $0.60-0.74=$ good agreement, ICC $0.75-1.0=$ very good agreement.

\section{Results}

\section{Radiation dose}

- Table 3 includes data regarding the applied radiation dose. For the 70-kV CT examinations, there was an average $\mathrm{CTDI}_{\text {vol }}$ value of $2.9 \pm 0.9 \mathrm{mGy}$, an average DLP of $99.9 \pm 31.0 \mathrm{mGy} \times \mathrm{cm}$, an average ED of $1.5 \pm 0.6 \mathrm{mSv}$ and an average SSDE of $3.8 \pm 1.2 \mathrm{mGy}$. In patients examined with a standard $120-\mathrm{kV}$ CT protocol, there was an average $\mathrm{CTDI}_{\text {vol }}$ value of $8.8 \pm 3.7 \mathrm{mGy}$, an average DLP of $296.6 \pm 119.3 \mathrm{mGy} \times \mathrm{cm}$, an average ED of $4.4 \pm 2.1 \mathrm{mSv}$ and an average SSDE of $11.6 \pm 4.4 \mathrm{mGy}$. The CTDI, DLP, ED and SSDE were significantly lower for high-pitch $70-\mathrm{kV}$ CT examinations compared to standard 120-kV CT examinations of the chest (all $P<0.001)$. In total, the ED could be reduced by an average of $66 \%$ by using 70-kV CT protocols ( $\triangleright$ Fig. 1 ).

\section{Objective image quality}

The results of the objective image quality analysis are shown in - Table 4. An average SNR of 3.7 \pm 1.1 and an average CNR of $1.4 \pm 0.8$ for the descending aorta were seen in the study population examined with the high-pitch 70-kV CT protocol. In comparison, the objective image parameters were non-significantly higher in the control group of standard 120-kV CT examinations with an SNR of $3.9 \pm 1.4$ and a CNR of $1.6 \pm 1.0(p=0.113$ and $p=0.156$, respectively). For the lung parenchyma, an SNR of $11.8 \pm 3.2$ and a CNR of $14.0 \pm 4.0$ were seen for the $70-\mathrm{kV}$ high-pitch CT examinations which was non-significantly lower than in the standard 120$k V$ examinations with an SNR of $12.8 \pm 3.9$ and CNR of $14.9 \pm 4.5$ $(p=0.082$ and $p=0.287$, respectively).

\section{Subjective image quality}

With respect to the cumulative subjective image quality, an average point value of $4.5 \pm 0.4$ for high-pitch $70-\mathrm{kV} \mathrm{CT}$ images and $4.7 \pm 0.3$ for $120-k V C T$ images was seen $(p=0.052)$. The subjective image noise in the study group with $70=k V C T$ examinations of the chest was considered worse with an average value of $3.0 \pm$

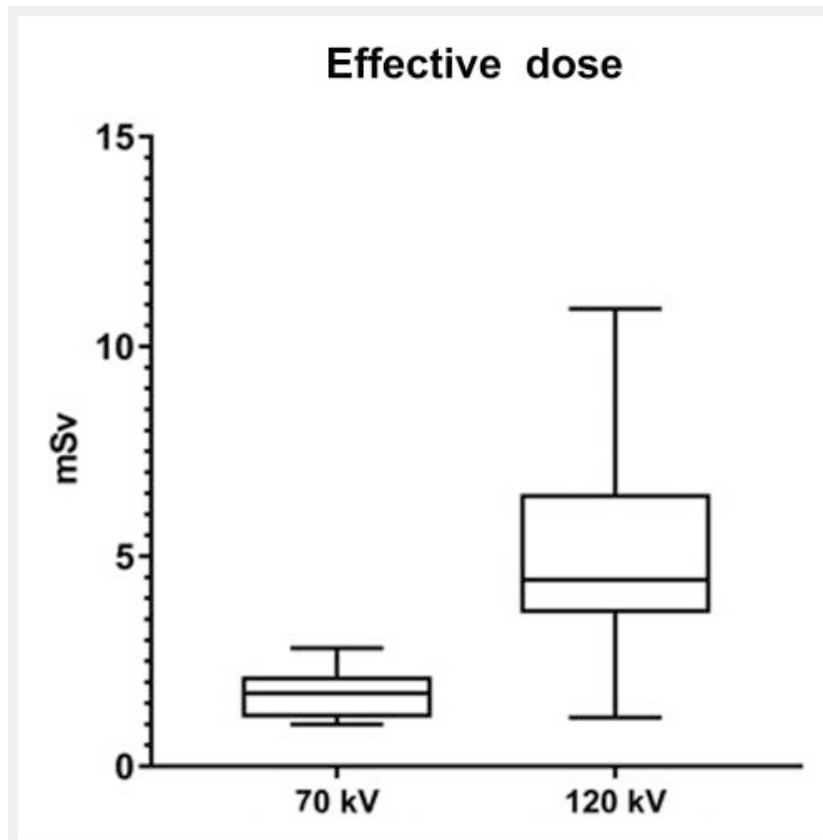

- Fig. 1 Effective dose values for the high-pitch 70-kV and standard 120-kV CT protocol. High-pitch 70-kV CT examinations of the thorax showed a significantly lower effective dose $(P<0.001)$.

0.4 vs. $3.5 \pm 0.6$ for $C T$ images using the $120-\mathrm{kV}$ standard protocol $(p=0.043)$. Similar results were observed for the comparison between $70-\mathrm{kV}$ and $120-\mathrm{kV}$ images regarding image sharpness (3.3 \pm 0.5 vs. $3.7 \pm 0.6 ; p=0.042$ ). However, high-pitch $70-k V$ examinations showed greatly reduced motion artifacts with consequently better ratings on the Likert scale ( $4.4 \pm 0.6$ vs. $3.8 \pm 0.9$; $\mathrm{p}=0.003$ ) ( $\triangleright$ Fig. 2a, b). However, similar mean values of $4.6 \pm 0.4$ for $70-k V$ and $4.7 \pm 0.3$ for $120-k V$ CT examinations without significant differences between the two groups $(p=0.829)$ were documented for both $\mathrm{CT}$ protocols with respect to subjective diagnostic reliability ( $\vee$ Fig. $3 a$, b).

The ICC analysis yielded good agreement between all three raters (general ICC $=0.72$ ). There was very good agreement regarding general image quality $(I C C=0.78)$ and the presence of motion artifacts (ICC $=0.75$ ), while the agreement regarding image noise $(I C C=0.70)$, image sharpness $(I C C=0.68)$, and subjec- 
- Table 4 Results of objective image analysis.

\begin{tabular}{|l|c|c|c|}
\hline & high-pitch 70-kV & standard 120-kv & p-value \\
\hline SNR $_{\text {aorta }}$ & $3.7 \pm 1.1$ & $3.9 \pm 1.4$ & $\mathrm{P}=0.113$ \\
\hline $\mathrm{SNR}_{\text {lung }}$ & $11.8 \pm 3.2$ & $12.8 \pm 3.9$ & $\mathrm{P}=0.082$ \\
\hline $\mathrm{SNR}_{\text {total }}$ & $7.8 \pm 2.1$ & $8.4 \pm 1.8$ & $\mathrm{P}=0.065$ \\
\hline $\mathrm{CNR}_{\text {aorta }}$ & $1.4 \pm 0.8$ & $1.6 \pm 1.0$ & $\mathrm{P}=0.156$ \\
\hline $\mathrm{CNR}_{\text {lung }}$ & $14.0 \pm 4.0$ & $14.9 \pm 4.5$ & $\mathrm{P}=0.287$ \\
\hline $\mathrm{CNR}_{\text {total }}$ & $7.7 \pm 2.4$ & $8.3 \pm 2.8$ & $\mathrm{P}=0.072$ \\
\hline $\mathrm{SNR}=$ signal-to-noise ratio; CNR $=$ contrast-to-noise ratio. & & \\
\hline
\end{tabular}

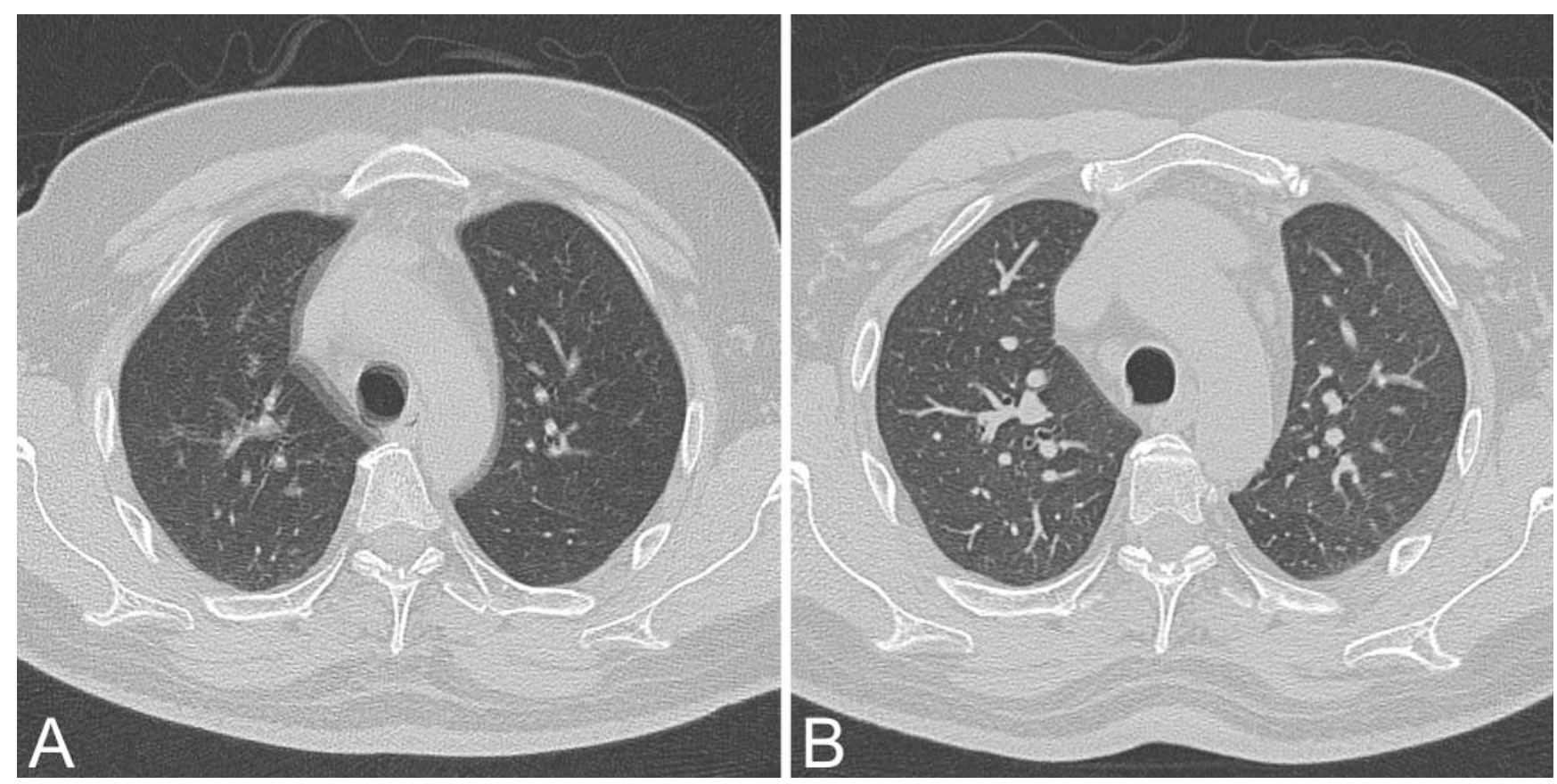

- Fig. 2 a Standard 120-kV chest CT of a 53-year-old patient after renal transplantation and neutropenic fever. This acquisition shows distinct movement artifacts due to breathing difficulties. b CT re-examination after 4 weeks because of acute dyspnea. The high-pitch 70-kV CT shows signs of pulmonary congestion. Despite breathing difficulties again, there are no movement artifacts.

tive diagnostic reliability $($ ICC $=0.71)$ was evaluated as good. - Table 5 provides the subjective image analysis results.

\section{Discussion}

The goal of our study was to evaluate an examination protocol with a reduced radiation dose for unenhanced CT examinations of the chest in immunosuppressed patients who require clinically indicated CT examinations and follow-ups due to their disease. Our study came to the conclusion that high-pitch 70-kV CT examinations offer a very good compromise between dose reduction and image quality and thus represent a very good alternative to the standard 120-kV CT protocol. Using the most recent imaging technology and the combination of high-pitch image acquisition and a reduced tube voltage, the radiation dose could be reduced in a targeted manner on average by two-thirds per examination. As a result, we were able to achieve a significantly lower effective patient dose with our study concept than Kubo et al. who developed a low-dose protocol by reducing the tube current [16]. Given a comparable objective image quality, there were fewer subjective assessments of image noise and image sharpness for $70-\mathrm{kV}$ imaging compared to $120-\mathrm{kV}$ CT. However, there was no significant difference in the subjective diagnostic reliability and cumulative subjective image quality in our study. However, according to Gordic et al., the increased image noise at $70 \mathrm{kV}$ could limit the detection of small pulmonary nodules [17]. The advantages of a lower tube voltage were able to be shown in earlier studies particularly in contrast-enhanced examinations since the iodine signal increases as the tube voltage decreases 

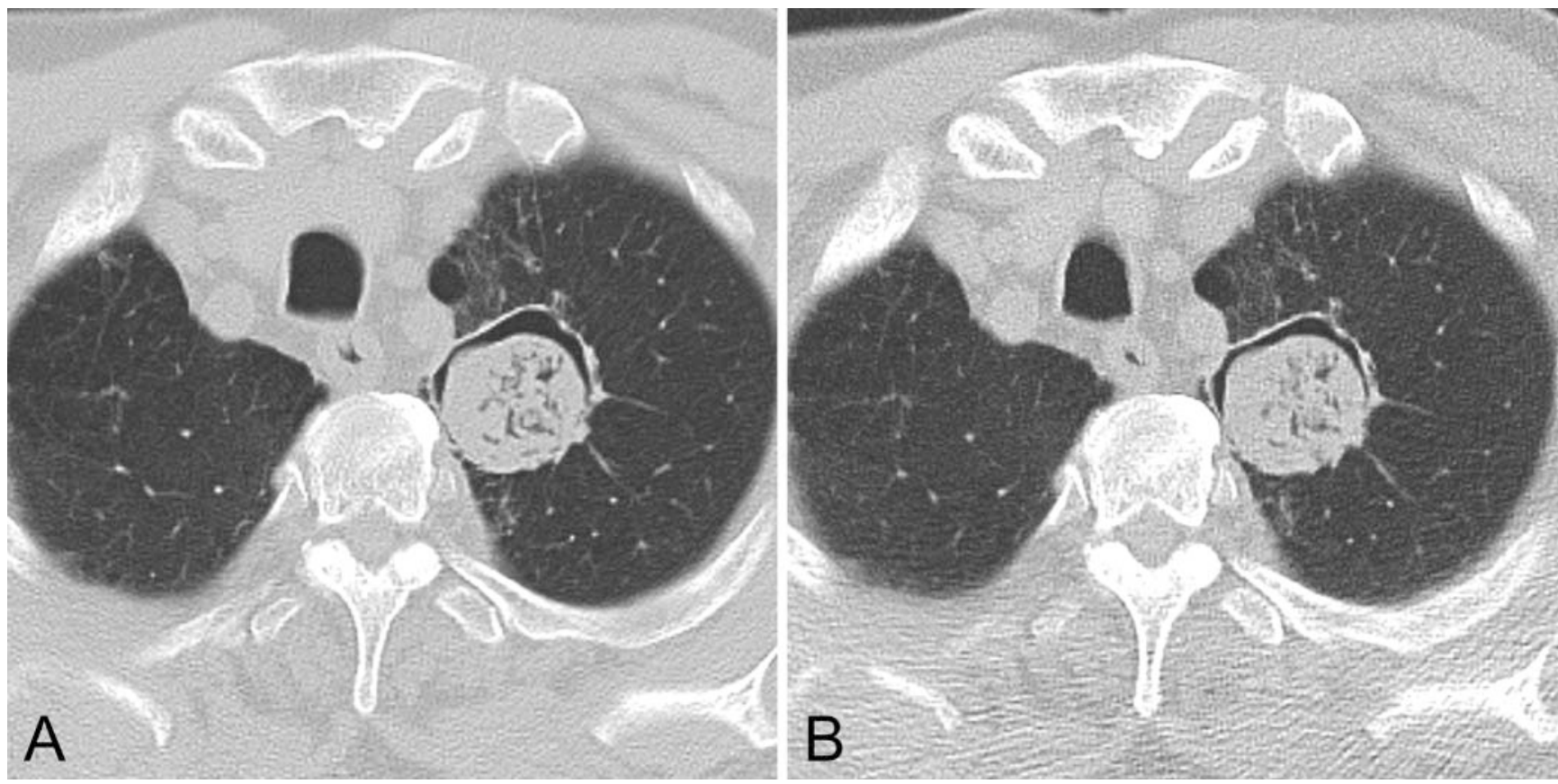

- Fig. 3 a Standard 120-kV chest CT of a 47-year-old patient with HIV and aspergilloma in the left apical upper lobe. b Follow-up examination after 2 months using the high-pitch 70-kV CT protocol. The aspergilloma is almost unchanged regarding size and appearance.

$[18,19]$. However, the results of our study show that the implementation of a 70-kV CT protocol has additional advantages even in the case of unenhanced examinations.

A high-pitch 80-kV chest CT protocol for pediatric patients without sedation or anesthesia was able to be successfully evaluated in a previous study by Bodelle et al. [20]. The authors were able to show a significant reduction in radiation dose with comparable image quality after reconstruction with ADMIRE. In general, modern low-dose CT imaging is always designed in combination with iterative image reconstruction and therefore is to be viewed as essential for this type of image acquisition [17, 21, 22]. In our study the lower radiation energy of the high-pitch 70 $\mathrm{kV}$ examinations with comparable objective image quality resulted in worsening of the subjective image noise and image sharpness despite reduction with ADMIRE as already described in previous studies [23-26]. However, this did not result in any limitations regarding the subjective diagnostic reliability. The high pitch factor probably had a supportive effect here. As a result of the greater table speed, $\mathrm{CT}$ examinations of the chest could be performed in less than two seconds [27]. There were consequently fewer motion artifacts (including breathing and pulsation artifacts), resulting in a higher cumulative image quality rating $[14$, 28]. Patients with respiratory issues benefited the most from this improvement in image quality [28]. Since immunosuppressed patients tend to undergo more frequent direct chest $\mathrm{CT}$ examinations than conventional $\mathrm{X}$-ray examinations in order to diagnose or rule out atypical pneumonia or other opportunistic infections of the lung, we feel that such dose-reducing measures are even more important in immunosuppressed patients than in immunocompetent patients since the effect can be even greater in the case of repeated imaging.
The present retrospective study has the following limitations. All examinations were performed on one third-generation dualsource CT scanner. Models from different manufacturers can vary with respect to design, technical specifications, and results. In this connection the restriction of the field-of-view (FOV) due to the use of the second tube and the smaller detector is a possible limitation of high-pitch image acquisition that can be relevant particularly for obese patients [29]. Moreover, the combination of tube voltage reduction and high-pitch technique does not allow indepth retrospective analysis of the effect of the individual factors on the resulting effective dose. Additional examinations with high-pitch $120-\mathrm{kV}$ and standard table speeds at $70-\mathrm{kV}$ would have to be performed for this purpose. Since these methods are already sufficiently established, a direct comparison of the individual techniques was not performed [30, 31]. The goal of this study was to compare high-pitch 70-kV examinations with the clinical standard to show the potential for the clinical routine. Moreover, the subjective diagnostic reliability was analyzed as part of quality assessment but the diagnostic accuracy for the detection of pathologies was not determined. This should be further evaluated in homogeneous patient populations with specific diseases in future studies. For the calculation of the ED (via conversion factor) and the SSDE, there was an approximation to $80-\mathrm{kV}$ CT examinations since there is insufficient data for $70-\mathrm{kV}$ examinations [11, 12, 32]. Moreover, only unenhanced CT images of the chest were examined in our study. Contrast-enhanced examinations were not evaluated in greater detail regarding objective and subjective image quality. However, contrast-enhanced imaging at $70 \mathrm{kV}$ could provide even better results due to the optimized contrast conditions. 
- Table 5 Results of subjective image analysis.

\begin{tabular}{|c|c|c|c|}
\hline & high-pitch 70-kV & standard $120-k V$ & p-value \\
\hline image quality & $4.5 \pm 0.4$ & $4.7 \pm 0.3$ & $p=0.052$ \\
\hline $\operatorname{ICC}(95 \% \mathrm{CI})$ & \multicolumn{2}{|l|}{$0.78(0.65-0.95)$} & \\
\hline image noise & $3.0 \pm 0.4$ & $3.5 \pm 0.6$ & $p=0.043$ \\
\hline ICC (95\% CI) & \multicolumn{2}{|l|}{$0.70(0.48-0.87)$} & \\
\hline image sharpness & $3.3 \pm 0.5$ & $3.7 \pm 0.6$ & $p=0.042$ \\
\hline ICC (95\% CI) & \multicolumn{2}{|l|}{$0.68(0.38-0.76)$} & \\
\hline motion artifacts & $4.4 \pm 0.6$ & $3.8 \pm 0.9$ & $p=0.003$ \\
\hline $\operatorname{ICC}(95 \% \mathrm{CI})$ & \multicolumn{2}{|l|}{$0.75(0.52-0.91)$} & \\
\hline diagnostic reliability & $4.6 \pm 0.4$ & $4.7 \pm 0.3$ & $p=0.829$ \\
\hline ICC (95\% CI) & \multicolumn{2}{|l|}{$0.71(0.51-0.90)$} & \\
\hline
\end{tabular}

\section{Conclusion}

Immunosuppressed patients could benefit from a significant dose reduction as a result of the use of a high-pitch $70-\mathrm{kV}$ chest $\mathrm{CT}$ protocol. There are no significant differences regarding objective and subjective image quality compared to standard 120-kV CT examinations. For this reason we recommend using a high-pitch 70-kV examination protocol particularly for CT follow-ups in immunosuppressed patients.

\section{CLINICAL RELEVANCE OF THE STUDY}

- High-pitch 70-kV CT examinations show no significant differences regarding objective and subjective image quality compared to standard $120-\mathrm{kV}$ CT examinations of the chest.

- Immunosuppressed patients could benefit from a significant dose reduction as a result of the use of a high-pitch 70-kV chest CT protocol.

- We recommend using a high-pitch 70-kV examination protocol particularly for $\mathrm{CT}$ follow-ups in immunosuppressed patients.

\section{Conflict of Interest}

Moritz H. Albrecht hat Referentenhonorare von Siemens erhalten. Julian L. Wichmann hat Referentenhonorare von Siemens und General Electric erhalten.

\section{References}

[1] Bajaj SK, Tombach B. Respiratory infections in immunocompromised patients: Lung findings using chest computed tomography. Radiology of Infectious Diseases 2017; 4: 29-37
[2] Memoli M], Athota R, Reed S et al. The Natural History of Influenza Infection in the Severely Immunocompromised vs Nonimmunocompromised Hosts. Clinical Infectious Diseases: An Official Publication of the Infectious Diseases Society of America 2014; 58: 214 - 224

[3] Köhler JR, Casadevall A, Perfect J. The Spectrum of Fungi That Infects Humans. Cold Spring Harbor Perspectives in Medicine 2015; 5: a019273

[4] Ewig S, Höffken G, Kern WV et al. S3-Leitlinie: Behandlung von erwachsenen Patienten mit ambulant erworbener Pneumonie und Prävention Update 2016. Deutschen Gesellschaft für Pneumologie und Beatmungsmedizin

[5] Brodoefel H, Vogel M, Hebart H et al. Long-term CT follow-up in 40 nonHIV immunocompromised patients with invasive pulmonary aspergillosis: kinetics of CT morphology and correlation with clinical findings and outcome. American journal of roentgenology 2006; 187: 404-413

[6] Campanella F, Rossi L, Giroletti E. Are physicians aware enough of patient radiation protection? Results from a survey among physicians of Pavia District- Italy. BMC health services research 2017; 17: 406

[7] Amis ES Jr., Butler PF, Applegate KE et al. American College of Radiology white paper on radiation dose in medicine. Journal of the American College of Radiology 2007; 4: 272 - 284

[8] Smith-Bindman R, Lipson J, Marcus R et al. Radiation Dose Associated with Common Computed Tomography Examinations and the Associated Lifetime Attributable Risk of Cancer. Archives of internal medicine 2009; 169: $2078-2086$

[9] Prakash P, Kalra MK, Ackman JB et al. Diffuse lung disease: CT of the chest with adaptive statistical iterative reconstruction technique. Radiology 2010; 256: 261 - 269

[10] Bongartz G, Golding S, Jurik A et al. European Guidelines on Quality Criteria for Computed Tomography. Report EUR 162621999

[11] Deak PD, Smal Y, Kalender WA. Multisection CT protocols: sex-and age-specific conversion factors used to determine effective dose from dose-length product. Radiology 2010; 257: 158-166

[12] Boone J, Strauss K, Cody D et al. Size-specific dose estimates (SSDE) in pediatric and adult body CT exams: Report of AAPM Task Group 204. 2011

[13] Martin SS, Albrecht MH, Wichmann JL et al. Value of a noise-optimized virtual monoenergetic reconstruction technique in dual-energy CT for planning of transcatheter aortic valve replacement. European radiology 2017; 27: $705-714$ 
[14] Bodelle B, Fischbach C, Booz C et al. Free-breathing high-pitch 80kVp dual-source computed tomography of the pediatric chest: Image quality, presence of motion artifacts and radiation dose. European journal of radiology 2017; 89: 208-214

[15] Cicchetti DV. Guidelines, criteria, and rules of thumb for evaluating normed and standardized assessment instruments in psychology. Psychological assessment 1994; 6: 284

[16] Kubo T, Ohno Y, Nishino M et al. Low dose chest CT protocol (50 mAs) as a routine protocol for comprehensive assessment of intrathoracic abnormality. European Journal of Radiology Open 2016; 3: 86-94

[17] Gordic S, Morsbach F, Schmidt B et al. Ultralow-dose chest computed tomography for pulmonary nodule detection: first performance evaluation of single energy scanning with spectral shaping. Investigative radiology 2014; 49: 465-473

[18] Wichmann JL, Hu X, Kerl JM et al. 70 kVp computed tomography pulmonary angiography: potential for reduction of iodine load and radiation dose. J Thorac Imaging 2015; 30: 69- 76

[19] Schueller-Weidekamm C, Schaefer-Prokop CM, Weber M et al. CT angiography of pulmonary arteries to detect pulmonary embolism: improvement of vascular enhancement with low kilovoltage settings. Radiology 2006; 241: 899-907

[20] Bodelle B, Klement D, Kerl JM et al. $70 \mathrm{kV}$ computed tomography of the thorax: valence for computer-assisted nodule evaluation and radiation dose - first clinical results. Acta radiologica (Stockholm, Sweden: 1987) 2014; 55: 1056 - 1062

[21] Newell JD Jr, Fuld MK, Allmendinger T et al. Very low-dose (0.15 mGy) chest $\mathrm{CT}$ protocols using the COPDGene 2 test object and a third-generation dual-source CT scanner with corresponding third-generation iterative reconstruction software. Investigative radiology 2015; 50: 40

[22] Schaller F, Sedlmair M, Raupach R et al. Noise Reduction in Abdominal Computed Tomography Applying Iterative Reconstruction (ADMIRE). Acad Radiol 2016; 23: 1230 - 1238

[23] Robertson DD, Weiss PJ, Fishman EK et al. Evaluation of CT techniques for reducing artifacts in the presence of metallic orthopedic implants. Journal of computer assisted tomography 1988; 12: 236-241
[24] Baumueller S, Winklehner A, Karlo C et al. Low-dose CT of the lung: potential value of iterative reconstructions. European radiology 2012; 22: $2597-2606$

[25] Tang K, Wang L, Li R et al. Effect of low tube voltage on image quality, radiation dose, and low-contrast detectability at abdominal multidetector CT: phantom study. Journal of biomedicine \& biotechnology 2012; 2012: 130169

[26] Weis M, Henzler T, Nance JW Jr et al. Radiation Dose Comparison Between $70 \mathrm{kVp}$ and $100 \mathrm{kVp}$ With Spectral Beam Shaping for NonContrast-Enhanced Pediatric Chest Computed Tomography: A Prospective Randomized Controlled Study. Investigative radiology 2017; 52: $155-162$

[27] Lell MM, May M, Deak P et al. High-pitch spiral computed tomography: effect on image quality and radiation dose in pediatric chest computed tomography. Investigative radiology 2011; 46: 116-123

[28] Baumueller S, Alkadhi $\mathrm{H}$, Stolzmann $\mathrm{P}$ et al. Computed tomography of the lung in the high-pitch mode: is breath holding still required? Investigative radiology 2011; 46: $240-245$

[29] Thomas C, Ketelsen D, Tsiflikas I et al. Dual-energy computed tomography: is there a penalty in image quality and radiation dose compared with single-energy computed tomography? Journal of computer assisted tomography 2010; 34: 309-315

[30] Lell MM, Scharf M, Eller A et al. Feasibility of Respiratory-gated Highpitch Spiral CT: Free-breathing Inspiratory Image Quality. Acad Radiol 2016; 23: 406-412

[31] Schulz B, Jacobi V, Beeres M et al. Quantitative analysis of motion artifacts in high-pitch dual-source computed tomography of the thorax. J Thorac Imaging 2012; 27: $382-386$

[32] Nakagawa M, Ozawa Y, Sakurai K et al. Image quality at low tube voltage $(70 \mathrm{kV})$ and sinogram-affirmed iterative reconstruction for computed tomography in infants with congenital heart disease. Pediatric radiology 2015; $45: 1472-1479$ 